\title{
Design of the Belt Driving Device of the Laminator
}

\author{
Zhao gang LI Yu jie ZHANG \\ Shenyang Academy of Instrumentation Science, Shenyang City, Liaoning Province, 110043 \\ 13940458233@163.com
}

\begin{abstract}
Keywords: Laminator, Belt Driving Device, Design
Abstract. The design of the belt transmission device of the lamination machine is carried out in the paper. The classification and power analysis of belt drive is introduced. The force analysis of belt drive is presented, the centrifugal force generated by the pulling force, drive force of the belt, baseline length and the initial tension of the belt, and the design of the power is analyzed. The axial stress of the belt is calculated. The roll tensioning device of the laminator is determined preliminarily.
\end{abstract}

\section{Introduction}

In the roll of the material on the drum, a tension device is need to prevent the large use of tension, in order to prevent that the membrane material is pulled too much, which will result in wrinkles. The belt wheel is selected for this tensioning mechanism.

\section{Analysis of Classification and Power of Belt Drive}

Belt drive into a flat belt drive, V-belt drive, ribbed belt, timing belt and so on. The belt drive is mainly played a preload effect, the required speed of the transmission is not great, so the use of relatively simple conditions. This article is selected to the ordinary flat belt. The losses of the belt drive include: slip loss, internal friction loss, adhesion to the pulley face, and side frictional losses of the V-belt wedge and exit troughs, loss of air resistance, and loss of bearing friction. In combination with the above losses, the efficiency of the belt drive is about $80 \%$ to $98 \%$, and the drive design[1] is selected according to the type of the belt.

\section{Force Analysis of Belt Drive}

\section{(1) the tension produced by the centrifugal force}

A separation of the body with the pulley on the contact arc with a small section of the arc length $d l$ is selected , as shown in Figure 1. The balance equation between the centrifugal force with the centrifugal force $d F_{N}$ and the centrifugal tension force $F_{c}$, is presented as follows:

$$
\begin{aligned}
& d F_{N}=2 F_{c} \sin \frac{d \alpha}{2} \\
& d F_{N}=d l \frac{q v_{2}}{r}=r d \alpha \frac{q v_{2}}{r}=q v_{2} d \alpha \\
& F_{c}=q v_{2}
\end{aligned}
$$

And $q$ - Mass, $\mathrm{kg} / \mathrm{m}$; v-band speed, $\mathrm{m} / \mathrm{s} ; \mathrm{r}$ - pulley radius, $\mathrm{m}$.

The centrifugal force of band is shown in figure 1 , and the analysis of driving force is shown in figure 2 . 
2.

The centrifugal force of band is shown in Fig. 1, and the analysis of driving force is shown in Fig.

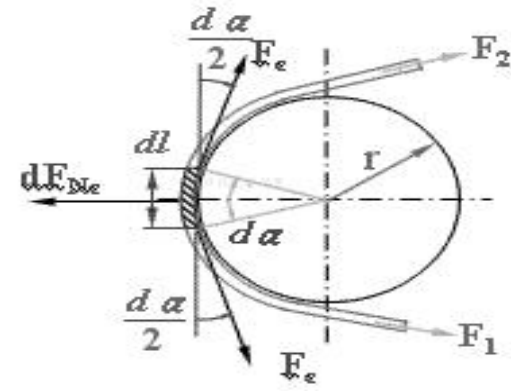

Figure 1. Centrifugal force of band

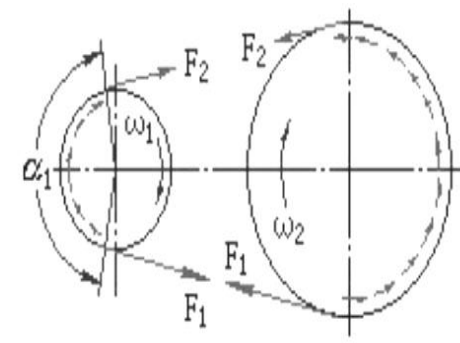

Figure 2. analysis of driving force

As the belt with the initial tension $F_{0}$ tension on the two pulleys, the role of $F_{0}$, with the pulley on equal, are $F_{0}$. The friction force $F_{f}$ of the driving wheel pair is consistent with the movement direction of the belt, and the friction force $F_{f}$ of the driven wheel pair is opposite to the movement direction of the belt. So the active side is tightened, the tension increased from $F_{0}$ to $F_{1}$, driven by the pull from $F_{0}$ reduced to $F_{2} . F_{1}$ is called tight side tension, $F_{2}$ is called loose edge tension.

$$
F_{1}-F_{0}=F_{0}-F_{2}
$$

As shown in Fig. 3, the belt wound around the capstan is separated, if the diameter of the capstan is $d_{d 1}$. The following equation is obtained according to the moment balance condition

$$
\sum T=0
$$

$$
\text { And } \begin{array}{r}
F_{f} \frac{d_{d 1}}{2}+F_{2} \frac{d_{d 1}}{2}-F_{1} \frac{d_{d 1}}{2}=0 \\
F_{f}=\sum F_{f i}=F_{e}=F_{1}-F_{2}
\end{array}
$$

In the equation, $F_{e}$-the effective circular force; $F_{f i}$ - the friction at each point.

$$
T_{1}=F_{f} \frac{d_{d 1}}{2}=F_{e} \frac{d_{d 1}}{2}
$$

The effective circumferential force $F_{e}$ is equal to the sum of the frictional forces at the points on the contact with the pulley, and is equal to the difference between the tension of the tight side and the loose edge.

The power that the belt drive can transmit is shown as follows:

$$
P=F_{e} v
$$

$F_{1}, F_{2}$ and the maximum effective circular force $F_{\text {emax }}$ are expressed as follows: a microstrip $d l$ long band is taken to study the microstrip band, the force balance equation in the $\mathrm{x}$ and $\mathrm{y}$ directions is shown as follows:

$$
\begin{gathered}
\sum x=0:(F+d F) \sin \frac{d \alpha}{2}+F \sin \frac{d \alpha}{2}-d N-d F_{N}=0 \\
\sum y=0:(F+d F) \cos \frac{d \alpha}{2}-F \cos \frac{d \alpha}{2}-f d N=0
\end{gathered}
$$

The high order infinitesimal are omitted while the two sides are integrated

$$
\frac{F_{1}-q v^{2}}{F_{2}-q v^{2}}=e^{f \alpha}
$$

$f$ - coefficient of friction; $\alpha$-wrap angle on small pulley 
In summary, the tension force for the tight side and the loose side for the critical state is presented as follows: .

$$
F_{1}=F_{e m a x} \frac{e^{f \alpha}}{e^{f \alpha}-1}+q v^{2} \quad F_{2}=F_{e m \text { ax }} \frac{1}{e^{f \alpha}-1}+q v^{2} \quad F_{e m \text { ax }}=2\left(F_{0}-q v^{2}\right) \frac{e^{f \alpha}-1}{e^{f \alpha}+1}
$$

From the above analysis the factors affecting the belt drive ability are discussed as follows: (1) the initial pull $F_{0} . F_{0}$ the greater the belt with the pulley will be the greater the pressure, the greater the $F_{\text {emax }}$. But $F_{0}$ is too large, with the wear and tear increased; with the internal stress increased band caused by relaxation, and life expectancy, if $F_{0}$ is too small, with the work capacity is not enough, easy to jump and slip when working. (2) Corner $\alpha$. The maximum effective circular force $F_{\text {emax }}$ increases with increasing $\alpha$. General requirements $\alpha_{\min } \geq 120^{\circ}$, special circumstances, allow $\alpha_{\min }=90^{\circ}$. (3) friction coefficient $f . f$ big $F_{\max }$ big. $f$ with the belt and pulley material, surface conditions, working conditions and so on. (4) the unit with the length of the quality of $q . q$ the greater the centrifugal force of the belt. So that $F_{\max }$ drops. Therefore, for high-speed belt drive, should choose a light quality with a high degree. (5) with speed $v \cdot v$ is larger, then the greater the centrifugal force of the belt, so that $F_{\max }$ drop more. General $v \leq 25 \mathrm{~m} / \mathrm{s}$.

\section{Calculation of Belt Drive}

\section{(1) Determine the reference length of the band $L_{d}$}

Pulley drive center distance selection: the center distance is small structure, but with too many short turns, will reduce the belt life, but also to $\alpha_{1}$ smaller, reducing the transmission capacity. The calculated length of the general band $L_{c}$ can be obtained according to the following geometric relations:

$$
\begin{aligned}
& 0.7\left(d_{1}+d_{2}\right) \leq a_{0} \leq 2\left(d_{1}+d_{2}\right) \\
\theta & =\arcsin \frac{d_{2}-d_{1}}{2 \alpha} \approx \frac{d_{2}-d_{1}}{2 \alpha} \\
L_{c}= & 2 \overline{A B}+\overparen{B C}+\overparen{A D}=2 a_{0} \cos \theta+\frac{1}{2}\left(d_{1} \alpha_{1}+d_{2} \alpha_{2}\right)
\end{aligned}
$$

The approximate formula is presented as followings:

$$
L_{c} \approx 2 a_{0}+\frac{\pi}{2}\left(d_{2}+d_{1}\right)+\frac{\left(d_{2}-d_{1}\right)^{2}}{4 a_{0}} 。
$$

\section{(2) Determine the center distance}

The center distance is calculated from the approximate formula

$$
a=a_{0}+\frac{L_{d}-L_{c}}{2}(\mathrm{~mm})
$$

Taking into account the installation, adjustment, compensation preload, the center distance from the need to have a certain range, of which the size of

$$
a_{\text {min }}=a-0.015 L_{d} ; \quad a_{\max }=a+0.03 L_{d}
$$


Take the diameter of the tension wheel $d_{1}=50 \mathrm{~mm}$, the diameter of the belt on the film $d_{2}=200 \mathrm{~mm}$. The film is pulled out at a rate of $50 \mathrm{~mm} / \mathrm{s}$. Find $a_{0}=400 \mathrm{~mm} ; \alpha_{2}=3.52 ; L_{c}=1210 \mathrm{~mm}$; reference length $L_{d}=1200 \mathrm{~mm}$. At the same time check the package angle $\alpha_{1}$ $\alpha_{1} \approx 180^{\circ}-\frac{d_{2}-d_{1}}{a} \times 60^{\circ}=157.8^{\circ} \geq 120^{\circ}$

Since the belt drive used is a tensioning device, the number of selected bands is one.

(3) Determine the initial tension and design power

Pull the required tension of the film material $F_{e \max }=20 \mathrm{~N}, \quad F_{e \max }=2\left(F_{0}-q v^{2}\right) \frac{e^{f \alpha}-1}{e^{f \alpha}+1}$

The first pull is obtained as $F_{0}, F_{0}=37.3 \mathrm{~N}$

According to the initial tension of a single band $F_{0}$ Calculation formula: $F_{0}=500 \frac{P_{c}}{v z}\left(\frac{2.5}{K_{a}}-1\right)+q v^{2}(\mathrm{~N})$, the design power is presented as: $P_{c} \approx 2.5 \mathrm{~W} ;$

The pressure on the axis $\mathrm{Q}$ is expressed as : $Q=2 F_{0} z \sin \frac{\alpha_{1}}{2}=73.2 \mathrm{~N}$

\section{Conclusions}

A preliminary analysis of the belt drive is carried out to determine the initial pulling force of the belt drive. And the diameter, angle and center distance of the pulley are calculated, and the force diagram and the formula derivation of the force caused by the centrifugal force of the belt drive and the transmission and other parameters is obtained, while determining the design power to calculate the pressure with the shaft. Then the film machine film tensioning device can be determined.

\section{Acknowledgements}

Thanks for the support of Shenyang Academy of Instrumentation Science.

\section{References}

[1] Gui Yuan Long, Yang Chun. Product Design. Beijing: China Light Industry Press, (2014)

[2] Yuan Juntao. A new type of printing, drying and film machine. Suzhou University, (2011)

[3] Peng HUANG. Design of Some Type of Mounter. Northeastern University,(2016)

[4] Wang Da Peng,Cheng Peng,Wan Jia. Design of automatic film machine for universal mobile phone screen. Academic Exchange,(2014) 8. 